\title{
Fast high-efficiency integrated waveguide photodetectors using novel hybrid vertical/butt coupling geometry
}

\author{
J. B. D. Soole, Y. Silberberg, A. Scherer, H. P. LeBlanc, \\ N. C. Andreadakis, and C. Caneau \\ Bellcore, 331 Newman Springs Road, Red Bank, New Jersey 07701 \\ $H$. Schumacher and U. Erben, \\ University of Ulm, W-7900 Ulm, Germany
}

(Received 12 March 1992; accepted for publication 7 May 1992)

\begin{abstract}
We report a novel coupling geometry for integrated waveguide photodetectors-a hybrid vertical coupling/butt coupling scheme that allows the integration of fast, efficient, photodetectors with conventional double heterostructure waveguides. It can be employed to yield a planar, or pseudo-planar, surface that supports further levels of integration. The approach is demonstrated with a $25-\mu \mathrm{m}$-long $p-i-n$ detector integrated with an InP/InGaAsP/ InP waveguide, which displays a high $(\sim 90 \%)$ efficiency and large $(\sim 15 \mathrm{GHz})$ bandwidth. This is the fastest high-efficiency integrated waveguide photodetector reported to date.
\end{abstract}

Waveguide photodetectors are key elements in integrated optoelectronics, allowing the monolithic integration of waveguide-based optical processing components and receiver electronics. ${ }^{1}$ To date, there have been two main approaches to achieve monolithic waveguide/detector integration-vertical coupling ${ }^{2-7}$ and butt coupling. ${ }^{8-12}$

In the vertical coupling geometry, the detector sits on top of the waveguide and light leaks up from the guide into the detector's absorption region. The fact that the vertically coupled detector sits proud of the surface, however, reduces surface planarity and increases the difficulty of obtaining small feature sizes in any integrated electronic or waveguide component. The length of detector needed to absorb most of the light power also tends to be rather large, ${ }^{2-5}$ and the resulting device capacitance can seriously limit receiver bandwidth and sensitivity. Significant length reductions may be obtained by growing a carefully designed intermediate coupling layer(s) between the guide and detector, ${ }^{6,7}$ however this technique is generally appropriate only for single heterostructure waveguides, and the use of a double heterostructure waveguide is precluded.

In the alternative butt coupling approach, guided light is end-fire coupled directly into the side, or end, of the photodetector. ${ }^{8-12}$ Either the detector material is regrown in a pit etched into the waveguide, ${ }^{8,11}$ or else the waveguide is regrown up against the wall of the detector mesa. $9,10,12$ The result is a planar or quasi-planar surface that is suitable for further integration. The butt-coupled detector needs only to be a few microns long in order to absorb all the guided light, and the small device capacitance that results allows it to be used in high bandwidth and high sensitivity receivers. Regrowth of the photodetector has been less popular, probably because of the difficulty of obtaining high quality refill growth of defect-free detector material. Regrowth of the waveguide structure, however, has yielded detectors with high quantum efficiencies, although to date there have been no reports of devices optimized for high speed. The unattractive feature of the conventional butt-coupled structure is that the complete waveguide structure must be regrown ${ }^{-}$throughout. In- creased growth rates near masked areas, ${ }^{13}$ such as the detector, lead to changes in mode profile and reduce the uniformity and control of the waveguiding. Most importantly, any imperfections in the regrowth have an immediate impact on the quality and integrity of the guiding.

In this letter we demonstrate a novel coupling scheme, which is a hybrid of the conventional vertical and butt coupling approaches. ${ }^{14}$ It combines the advantages of cach, while avoiding their respective drawbacks. In it, the waveguide core runs continuously underneath the detector, as for the vertical coupling, but the guide cladding layer is regrown up against the detector, as in the butt coupling case; see Fig. 1. The result is a strong coupling of the light between the waveguide and detector, allowing short detectors to absorb all the incident waveguide light. By growing a cladding layer of similar thickness to the detector, a planar structure may be produced which supports further degrees of monolithic integration. Although regrowth is required, only the upper guide cladding is regrown and any growth rate modification or growth imperfection has a negligible effect on the mode propagation; the critical guidecore layer is already present. We illustrate this novel hybrid coupling geometry with a $25-\mu \mathrm{m}$-long $p-i-n$ detector, integrated with an InP/InGaAsP/InP waveguide. This short detector is seen to absorb almost all of the guided light ( $\sim 90 \%)$ and to have a bandwidth of $\sim 15 \mathrm{GHz}$ - the highest value recorded to date for a waveguide integrated detector.

The waveguide-detector structure was grown by low pressure (76 Torr) organometallic chemical vapor deposition (OMCVD) at $625^{\circ} \mathrm{C}$ on semi-insulating InP. A semiinsulating substrate was chosen, rather than an $n^{+}$substrate, because the former is needed if a monolithically integrated high bandwidth receiver is to be realized.

First, the waveguide core and detector layers were grown. The guide core was $0.6 \mu \mathrm{m}$ of undoped InGaAsP $\left(\lambda_{g}=1.1 \mu \mathrm{m}\right)$ on a $0.5 \mu \mathrm{m}$ InP buffer layer. The detector comprised a $0.1 \mu \mathrm{m} n$-InP $\left(3-510^{18} \mathrm{~cm}^{-3}\right)$ contact layer, a $0.7 \mu \mathrm{m}$ undoped InGaAs absorption layer, $0.4 \mu \mathrm{m}$ of $p$-InGaAsP $\left(\lambda_{g}=1.3 \mu \mathrm{m}\right)\left(0.2 \mu \mathrm{m}\right.$ at $1 \times 10^{17} \mathrm{~cm}^{-3}$, fol- 


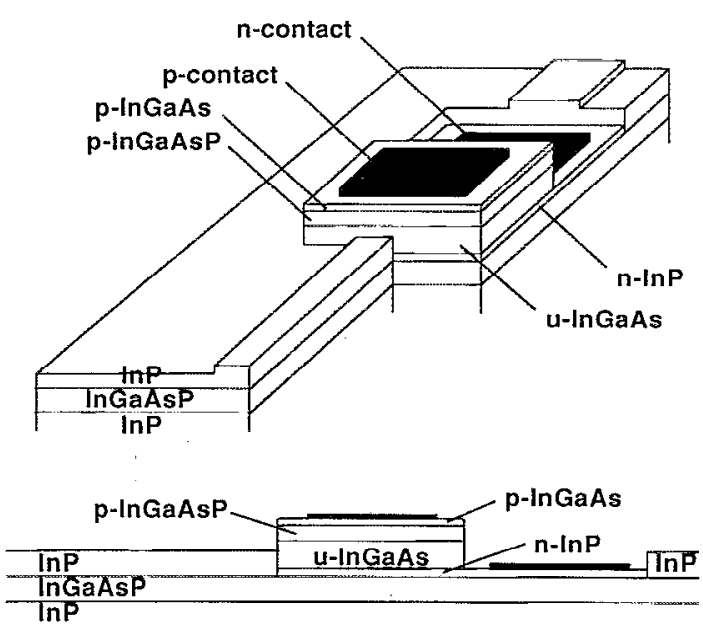

FIG. 1. Schematic of the waveguide detector employing hybrid vertical/ butt coupling. The $p-i-n$ detector sits on top of the waveguide core and is butt-joined to the guide cladding layer.

lowed by $0.2 \mu \mathrm{m}$ at $5 \times 10^{17} \mathrm{~cm}^{-3}$ ) and, finally, a $0.1 \mu \mathrm{m}$ $p^{+}$-InGaAs $\left(5 \times 10^{18} \mathrm{~cm}^{-3}\right)$ top contact layer.

Detector mesas were etched down to the top surface of the $\lambda_{\mathrm{g}}=1.1 \mu \mathrm{m}$ InGaAsP guide-core, using a $\mathrm{SiO}_{2}$ mask and a combination of dry and selective wet chemical etching techniques. The substrate was then returned to the OMCVD reactor for regrowth of the upper guide cladding layer on the unmasked guide-core; $0.5 \mu \mathrm{m}$ of undoped InP.

Fabrication of the waveguide detectors then proceeded with the etching of $5-\mu \mathrm{m}$-wide, $\sim 0.25-\mu \mathrm{m}$-deep, waveguide ridges into the InP cladding, which ran into the detectors along [110]. The $\mathrm{SiO}_{2}$ detector mask was then removed and a uniform $\mathrm{SiO}_{2}$ coating deposited, to provide good contact pad isolation for on-chip probing. The $p-i-n$ diodes were then fully processed. First, the oxide from the downstream half of the $50-\mu \mathrm{m}$-long $p-i-n$ mesas was removed and the mesa was etched down to the $n$-InP contact layer with a selective chemical etch $\left(\mathrm{H}_{3} \mathrm{PO}_{4} / \mathrm{H}_{2} \mathrm{O}_{2} / \mathrm{H}_{2} \mathrm{O}\right.$ at $1: 1: 8)$. Then, a polyimide isolation layer was deposited and windows were opened up for the contacts, the oxide on the mesa roof opening being removed to allow $p$ contacting. Finally, $n$ and $p$ contacts were formed in separate lift-olf metallizations ( $n$ contact using $\mathrm{Ni} / \mathrm{Ge} / \mathrm{Au} / \mathrm{Ni} / \mathrm{Au}$, [75 Å/ $350 \AA / 500 \AA / 350 \AA / 1500 \AA]$ and $p$ contact using $\mathrm{Pd} /$ $\mathrm{AuGe} / \mathrm{Au},[200 \AA / 800 \AA / 1800 \AA]$, and annealing. The metallizations ran to pads that were compatible with high speed on-chip probing. The InGaAs $p-i-n$ mesas thus formed, were $15 \mu \mathrm{m}$ wide (i.e., perpendicular to the input guides) and $25 \mu \mathrm{m}$ long; see Fig. 1 .

The chip was thinned to $\sim 150 \mu \mathrm{m}$ and cleaved perpendicular to the input guides. There was $0.8 \mathrm{~mm}$ of input guide before the detectors, with $1.2 \mathrm{~mm}$ after them. The detectors were fabricated in a dense one-dimensional array, perpendicular to the waveguides, separated by just $\sim 40$ $\mu \mathrm{m}$ and interspersed with guides without detectors on them.

The detectors were examined with $1.55 \mu \mathrm{m}$ wavelength light, end-fire coupled into the guides through a 0.25 N.A.

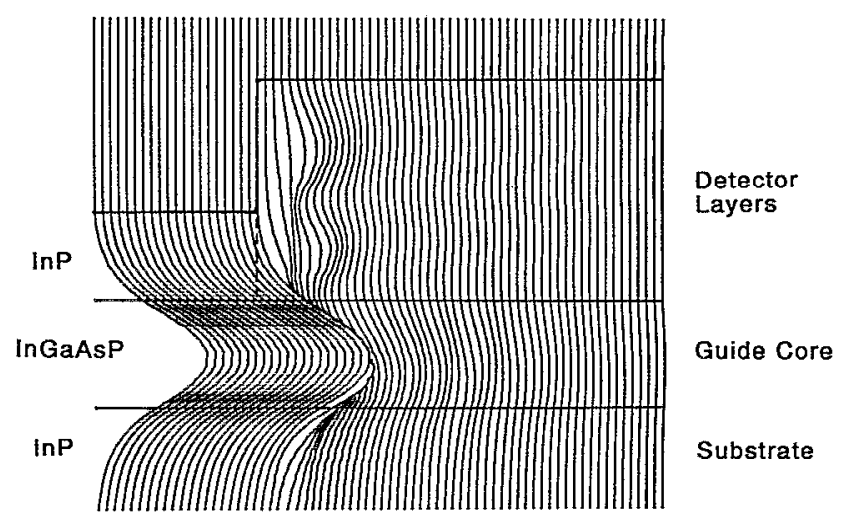

FIG. 2. BPM simulation of the wave-field profile (TE polarized light) as the guided mode passes underneath the detector structure. Field intensity $\left|E^{2}\right|$ is plotted for every $1 \mu \mathrm{m}$ of propagation. There is strong attenuation in the detector region and negligible radiative loss generated by the waveguide/detector interface.

microscope objective; polarization was selected to launch TE guide modes. Focusing the near-field image of the output facet of the detector-less guides onto an infrared vidicon showed the guides to be single moded and to provide high quality guiding. The dark current characteristics of the waveguide detectors were typical of similar sized (normally illuminated) mesa-etched $p-i-n$ 's, with dark currents of $\sim 10 \mathrm{nA}$ at $-5 \mathrm{~V}$ and breakdown above $10-15 \mathrm{~V}$.

Figure 2 shows a beam propagation method (BPM) simulation of the guided wave propagation through the waveguide/detector structure fabricated. The strong attenuation is apparent, and is plotted in Fig. 3. It indicates that a $25 \mu \mathrm{m}$ length of detector attenuates the guided mode by $85 \%$ ( $\pm 3 \%$ from refractive index uncertainties and device length tolerance). $95 \%$ absorption occurs after $\sim 42 \mu \mathrm{m}$. Negligible radiation is apparent from Fig. 2, and negligible absorption is computed for the upper $p$-contact layer and metallization. The light attenuation thus represents the ab-

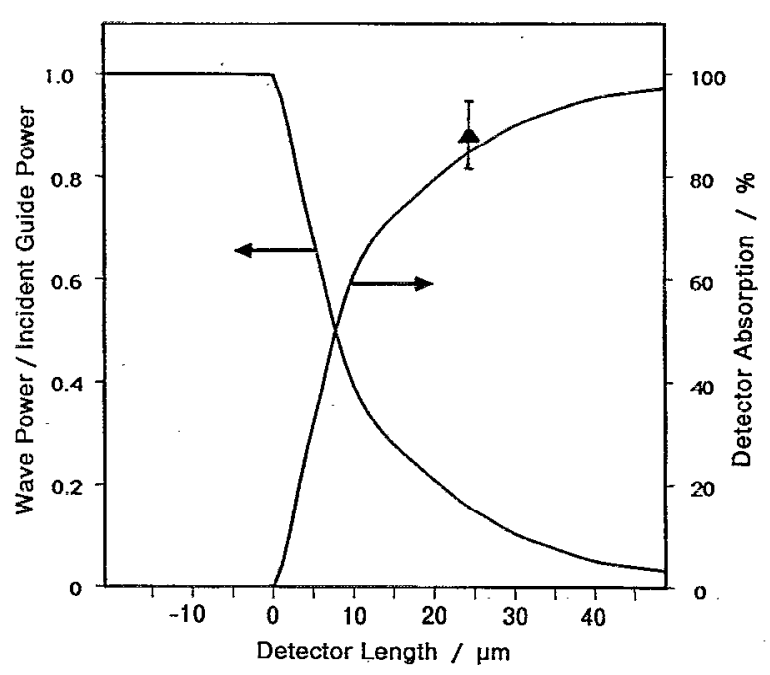

FIG. 3. Attenuation of the guided light simulated in Fig. 2. Detector absorption is plotted as [1-attenuation], assuming other losses to be negligible, and is equal to the efficiency of the waveguide detector. The experimentally observed value of $88 \pm 7 \%$ is also plotted. 


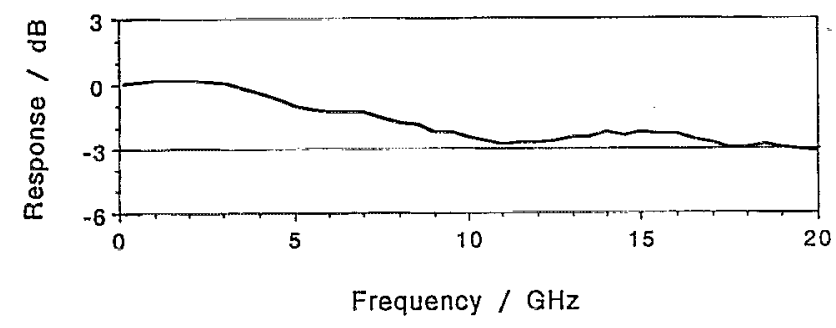

FIG. 4. Frequency response of the $25-\mu \mathrm{m}$-long detector.

sorption occurring in the InGaAs layer. This absorption is also plotted, as [1-attenuation], in Fig. 3 and corresponds to the predicted detector efficiency.

The experimental waveguide detector efficiency was determined from the photocurrent and the incident guide mode power, this latter being determined from the light transmitted by adjacent detector-less guides. For this purpose the output facet was antireflection coated. A small correction was made for the loss in the extra portion of the guide beyond the detectors.

The efficiency of the waveguide detectors was found to be $88 \pm 7 \%$; this value is also plotted in Fig. 3. The experimental uncertainty derives from the scatter in the recorded measurements (identical input coupling is required for each guide) and the difficulty of obtaining accurate absolute values for the power transmitted by the detectorless guides. The experimental value is nevertheless in very good agreement with the BPM calculation for a detector length of $25 \mu \mathrm{m}$, and confirms that almost all of the incident guide power is absorbed by the waveguide detector.

The light transmitted beyond the detectors was found to be $8 \% \pm 1 \%$ of the incident guide power. Though BPM simulations of the complete detector structure indicate a slightly higher value of $11.5 \% \pm 3 \%$, if we assume $12 \%$ transmission beyond the absorption region, in accord with the observed photocurrent, a figure of $9 \%$ is indicated, which is in good agreement with experiment.

The high value of the measured detector efficiency $(88 \%)$ confirms that the hybrid vertical/butt coupling geometry employed here is an effective means of obtaining a high absorption in a short length of detector.

The frequency performance of the waveguide detector was measured in the frequency domain using a $(0.13$ $\mathrm{MHz}-20 \mathrm{GHz}$ ) HP8703A lightwave component analyzer, and probing on-chip with a high bandwidth $(0-26.5$ GHz) Cascade Microtech probe. A typical result, with the lead, bias tee, and probe response calibrated out, is shown in Fig. 4. The slight peaking at $\sim 16 \mathrm{GHz}$, together with the preceding trough, are believed to be remaining measurement parasitics caused by residual reflections within an angled adaptor connected to the probe head. From a logarithmic plotting, we estimate the true diode $-3 \mathrm{~dB}$ bandwidth to be $15 \pm 0.5 \mathrm{GHz}$, rather than the higher frequency crossover point indicated in the figure. This is the highest bandwidth recorded for a waveguide-integrated photodetector to date. It is particularly noteworthy that this is achieved on a semi-insulating substrate, which permits the monolithic integration of receiver electronics.

The detector response is $\mathrm{RC}$ limited, carrier transit times across the $0.7 \mu \mathrm{m}$ depletion region being negligible in comparison to the $\mathrm{RC}$ charging time. ${ }^{15}$ The geometric capacitance of the $15 \mu \mathrm{m} \times 25 \mu \mathrm{m}$ detector is just $51 \mathrm{~F}$. The bandwidth limitation arises from the significant series resistance, measured from the forward bias characteristic to be in the range 120-135 $\Omega$. Fitted $S_{11}$ measurements indicated a series resistance of $\sim 140 \Omega$, together with a parasitic shunt capacitance of $\sim 12 \mathrm{fF}$, attributable to bond pads and interconnection lines. The series resistance is believed to arise largely from the contacts, with some contribution also from the $n$-InP sheet resistance and metal lines. With increased contact conductances and a somewhat thicker and more highly doped contact layer, we believe that bandwidths in excess of $30 \mathrm{GHz}$ are attainable without significant loss of power efficiency.

In summary, a novel geometry for obtaining effective light coupling between a waveguide and photodetector was demonstrated using a $25 \mu \mathrm{m}$ long $p-i-n$ detector. It recorded a $\sim 90 \%$ quantum efficiency and $\sim 15 \mathrm{GHz}$ bandwidth. Bandwidths in excess of $30 \mathrm{GHz}$ should be attainable with minor modifications. The planar device structure will facilitate higher levels of monolithic integration.

We wish to thank W. K. Chan and J. R. Hayes for helpful discussions, P. J. Rickmann for graphic assistance, and $\mathrm{W}$. Sessa for loan of the lightwave analyzer.

${ }^{1}$ T. L. Koch and U. Koren, IEEE J. Lightwave Technol. 3, 641 (1991).

${ }^{2}$ C. Bornholdt, W. Doldissen, F. Fiedler, R. Kaiser, and W. Kowalski, Electron. Lett. 23, 2 (1987).

${ }^{3}$ S. Chandrasekhar, J. C. Campbell, A. G. Dentai, and G. J. Qua, Electron. Lett. 23, 501 (1987).

${ }^{4}$ M. Erman, P. Jarry, R. Gamonal, J.-L. Gentner, P. Stephan, and C. Guedon, J. Lightwave Technol. 6, 399 (1988).

${ }^{5}$ J. B. D. Soole, H. Schumacher, R. Esagui, H. P. LeBlanc, R. Bhat, and M. A. Koza, Appl. Phys. Lett. 55, 2173 (1989).

${ }^{6}$ R. J. Deri, and O. Wada, Appl. Phys. Lett. 55, 2712 (1989).

${ }^{7}$ R. J. Deri, W. Doldissen, R. J. Hawkins, R. Bhat, J. B. D. Soole, L. M. Schiavone, M. Seto, N. Andreadakis, Y. Silberberg, and M. A. Koza, Appl. Phys. Lett. 58, 2749 (1991).

${ }^{8}$ G. E. Stillman, C. M. Wolfe, and I. Melngailis, Appl. Phys. Lett. 25, 36 (1974).

${ }^{9}$ S. Chandrasekhar, J. C. Campbell, A. G. Dentai, C. H. Joyner, G. J. Qua, and W. W. Snell, IEEE Electron. Dev. Lett. 8, 512 (1987).

${ }^{10}$ S. Chandrasekhar, J. C. Campbell, F. G. Storz, A. G. Dentai, C. H. Joyner, and G. J. Qua, Electron. Lett. 24, 1458 (1988).

${ }^{11}$ W. Doldissen, F. Fiedler, R. Kaiser, and L. Morl, Electron. Lett. 25, 35 (1989).

${ }^{12}$ J. B. D. Soole, H. Schumacher, H. P. LeBlanc, R. Bhat, and M. A. Koza, Appl. Phys. Lett. 56, 1518 (1990).

${ }^{13}$ E. Colas, A. Shahar, J. B. D. Soole, W. J. Tomlinson, J. R. Hayes, C. Caneau, and R. Bhat, J. Cryst. Growth 107, 226 (1991).

${ }^{14}$ J. B. D. Soole, H. Schumacher, H. P. LeBlanc, and R. Bhat, Technical Digest, IEEE/LEOS Topical Meeting on Integrated Optoelectronics, Monterey, July 1990 (IEEE, New York, 1990), pp. 48-49.

${ }^{15}$ J. E. Bowers and C. A. Burrus, IEEE J. Lightwave Technol. LT-5, 1339 (1987). 